Utah State University

DigitalCommons@USU

\title{
Confirmatory Factor Analysis and Measurement Invariance of the Cognitive Fusion Questionnaire-Body Image in a Clinical Eating Disorder Sample
}

\author{
Jennifer L. Barney \\ Utah State University, jen.barney4@aggiemail.usu.edu \\ Tyson S. Barrett \\ Utah State University, tyson.barrett@usu.edu \\ Tera Lensegrav-Benson \\ Avalon Hills Eating Disorder Specialists \\ Benita Quakenbush \\ Avalon Hills Eating Disorder Specialists \\ Michael P. Twohig \\ Utah State University, michael.twohig@usu.edu \\ Follow this and additional works at: https://digitalcommons.usu.edu/psych_stures \\ Part of the Psychology Commons
}

\section{Recommended Citation}

Barney, J. L., Barrett, T. S., Lensegrav-Benson, T., Quakenbush, B., \& Twohig, M. P. (in press). Confirmatory factor analysis and measurement invariance of the cognitive fusion questionnaire-body image in a clinical eating disorder sample. Body Image.

This Article is brought to you for free and open access by the Psychology Student Works at DigitalCommons@USU. It has been accepted for inclusion in Psychology Student Research by an authorized administrator of DigitalCommons@USU. For more information, please contact digitalcommons@usu.edu.

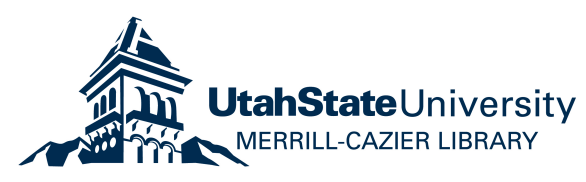




\title{
Confirmatory Factor Analysis and Measurement Invariance of the Cognitive Fusion Questionnaire-Body Image in a Clinical Eating Disorder Sample
}

\author{
Jennifer L. Barney, M.S. ${ }^{\text {a }}$ \\ Tyson S. Barrett, Ph.D. ${ }^{\mathrm{a}}$ \\ Tera Lensegrav-Benson, Ph.D. ${ }^{b}$ \\ Benita Quakenbush, Ph.D. ${ }^{\text {b }}$ \\ Michael P. Twohig, Ph.D. ${ }^{\text {a }}$ \\ ${ }^{\text {a }}$ Department of Psychology, Utah State University 2810 Old Main Hill, Logan UT 84322 USA \\ ${ }^{\mathrm{b}}$ Avalon Hills Eating Disorder Specialists, 175 E 100 N, Logan, UT 84321 USA
}

Corresponding author:

Jennifer L. Barney

Department of Psychology

Utah State University

2810 Old Main Hill

Logan, UT 84322

Office: (435) 797-8303

Email: jen.barney4@aggiemail.usu.edu 


\begin{abstract}
Individuals with eating disorders (EDs) may be particularly susceptible to body image related cognitive fusion (i.e., excessive entanglement with one's body image related thoughts such that they unduly influence on behavior). The Cognitive Fusion Questionnaire-Body Image (CFQ-BI) is the only existing measure of this construct, yet its psychometric properties have not been examined within a clinically diagnosed ED sample. The current study used confirmatory factor analysis and explored measurement invariance, construct validity, and incremental validity of the CFQ-BI when used with adolescent $(n=75)$ and adult $(n=100)$ females admitting to residential ED treatment. A modified version of the single-factor structure of the CFQ-BI best fit the data and configural, metric, and scalar invariance were supported across age groups (i.e., adolescents or adults) and ED behavioral presentations (i.e., restrictive behaviors or binge/purge behaviors). Adults' and individuals with binge/purge behavioral presentations reported significantly more body image related fusion compared to adolescents and adults, respectively. Body image related psychological flexibility and ED severity were both significantly correlated CFQ-BI scores in expected directions. These findings suggest the CFQ-BI is a valid measure for assessing body image related fusion among adolescent and adult females with varying ED behavioral presentations. Implications and future research directions are discussed.
\end{abstract}




\section{Keywords:}

Cognitive Fusion, Body Image, Eating Disorders, Factor Analysis 


\section{Introduction}

There is burgeoning empirical support for cognitive-behavioral theories (CBTs) of emotional disorders which posit that internal processes (e.g., thoughts, feelings, physical sensations) can influence behavioral change (Hofmann et al., 2012; Hofmann \& Hayes, 2018). However, variations in response rate to specific CBT methods at both the individual and disorder specific level highlight the importance of identifying when and why specific cognitive and behavioral processes specifically influence varying forms of psychopathology (Hofmann \& Hayes, 2018). Specifically, psychologists and clinicians have sought to elucidate what cognition processes appear most influential in the development and maintenance of various dysfunctional behavioral patterns to create targeted psychotherapy interventions to address them. To date, several cognitive processes have been theorized to be transdiagnostic contributors to psychopathology in this regard including irrational misappraisal (Barlow et al., 2004), repetitive negative thinking (McEvoy et al., 2013), and interpretative reasoning or expectancy reasoning biases (Morris \& Mansell, 2018).

A cognitive process more recently theorized to contribute to a wide array of psychopathology is cognitive fusion (Hayes et al., 2011). Cognitive fusion is defined as the degree to which an individual becomes entangled with the specific content of cognitions (e.g., thoughts, memories, assumptions, beliefs, and images), responding to them as literally true. In other words, one perceives their thoughts to be their reality, rather than seeing them simply as mental events, and responds behaviorally based on the content of the thought being true (Gillanders, et al. 2014). For example, if an individual were fused with the thought "I am stupid," they would take this literally, as an inarguable truth about themselves, and may put little effort into learning endeavors, even if doing so is inconsistent with their personal values. 
Elevated levels of cognitive fusion has been found to be predictive of various forms of psychopathology (Gillanders et al., 2014; Krafft et al., 2019; Pinto-Gouveia et al., 2020) and psychological interventions such as Acceptance and Commitment Therapy (ACT) have developed an array of strategies to specifically target cognitive defusion in response (Hayes et al., 2011).

Recent conceptual frameworks of eating disorders (EDs) have been developed which posit that cognitive fusion may play an integral role in the development and maintenance of disordered eating behavior (Merwin et al., 2010; Trindade \& Ferreira, 2014). Specifically, ACT based conceptualizations of EDs postulate that individuals with EDs may experience elevated levels of cognitive fusion with distressing thoughts about food, weight, and shape. For example, an individual with an ED may be fused to the thought "My life would be better if I was thinner," rigidly using this thought to guide their behaviors. In response, the "fused" individual may engage in a host of disordered eating behaviors such as dietary restriction, compulsive exercise, or purging even when doing so conflicts with direct contingencies (e.g., bodily cues of hunger/fullness; medical complications) or with personal values (e.g. interpersonal relationships; physical health; Manlick et al., 2013). Within this conceptualization, ACT theorizes that it is not the content of the thought that is problematic, but the fused relationship one has with the thought such that it dictates behavior without consideration of additional contextually relevant sources of information that may be present.

Much of the research to date examining the relationship between cognitive fusion and ED pathology has focused specifically on body image-related cognitive fusion as assessed by the Cognitive Fusion Questionnaire-Body Image (CFQ-BI; Ferreira et al., 2015). The CFQ-BI was developed by adapting items from a validated measure of general cognitive fusion (the Cognitive 
Fusion Questionnaire; Gillanders et al., 2014) to reflect levels of fusion with thoughts specifically regarding one's physical appearance. The original development and validation of the CFQ-BI produced a 10-item, single factor measure that has demonstrated high internal consistency within diverse samples including middle school and university student samples (Ferreira et al., 2015), individuals diagnosed with clinically significant Binge Eating Disorder (BED; Duarte et al., 2017), and overweight/obese individuals seeking weight loss treatment (Lucena-Santos et al., 2017). The CFQ-BI also demonstrated good incremental validity when compared to an overall measure of cognitive fusion in predicting self-reported ED symptoms, and scores were able to adequately discriminate between females who self-reported severe eating difficulties and those who did not (Ferreira et al., 2015). Further, the CFQ-BI demonstrated good internal reliability and temporal consistency when examined with a sample of male and female students (Ferreira et al., 2015) and had good convergent validity with measures of general cognitive fusion, psychological inflexibility, and characteristics of dispositional mindfulness/decentering (Ferreira et al., 2015; Lucena-Santos et al., 2017).

Findings from studies using the CFQ-BI have consistently shown that high levels of body image-related cognitive fusion are strongly associated with elevated global ED symptomology (Ferreira et al., 2014; Trindade \& Ferreira, 2014) and binge eating severity (Duarte et al., 2017; Lucena-Santos et al., 2017). Conversely, elevated CFQ-BI scores were found to be negatively associated with overall psychological health and quality of life (Ferreira \& Trindade, 2015). Mediation analyses have also provided initial evidence supporting the hypothesis that body image-related cognitive fusion appears to be a mechanism through which ED symptomology emerges and/or is maintained. Specifically, studies have found that scores on the CFQ-BI appear to mediate the relationship between ED symptomology and common risk factors for ED 
development such as body dissatisfaction (Trindade \& Ferreira, 2014), perceived discrepancies between one's real and ideal body size (Bento et al., 2017), and experiences of shame (Duarte \& Pinto-Gouveia, 2017; Duarte et al., 2017).

Although these findings provide initial support for the hypothesized role of body imagerelated cognitive fusion in eating pathology, there are notable limitations in their generalizability and clinical relevance. First, the CFQ-BI has not yet been validated within a sample of individuals diagnosed with clinically significant ED pathology. While the initial validation study does attempt to examine the CFQ-BI's ability to discriminate between individuals with and without clinically significant eating pathology, analyses are based solely on self-report data using suggested cut off scores on a commonly used screening tool for ED symptomology as opposed to clinical diagnosis (Ferreira et al., 2015). Lucena-Santos et al. (2017) also examined the psychometric properties and the ability of CFQ-BI scores to predict binge eating severity within a "clinical sample," however all participants in the study were obese or overweight individuals seeking weight-loss treatment. While reliable associations are observed between BED and overweight/obesity populations, the majority of individuals who experience overweight/obesity do not engage in recurrent binge eating and report significantly less functional impairment, subjective distress, and diminished quality of life relative to individuals who meet diagnostic criteria for BED (American Psychiatric Association, 2013). Existing research also fails to provide insight as to potential differences in body image-related cognitive fusion across ED diagnoses or individuals who present with different ED behavior profiles (i.e., EDs characterized by restrictive behaviors or EDs characterized by binging and/or purging behaviors). Finally, all but one study (Scardera et al., 2020) to our knowledge using the CFQ-BI to evaluate body image-related cognitive fusion in relation to eating pathology have been conducted by members 
of the same research team and primarily utilize samples from Portugal with a limited range of demographic characteristics (e.g., female, students or young adults from the general public, Portuguese speaking).

The current study therefore sought to examine the psychometric properties and proposed one-factor structure of the CFQ-BI within an English-speaking sample of adolescent and adult females diagnosed with clinically significant EDs admitting to residential treatment. We also sought to evaluate its convergent and incremental validity with theoretically relevant variables (i.e., body image related psychological flexibility and ED symptom severity). Finally, we conducted exploratory analyses to examine measurement invariance of the CFQ-BI when utilized with adolescent relative to adult patients, as well as between differing ED behavioral profiles (i.e., characterized by restrictive behaviors only or characterized by binging and/or purging behaviors). Based on previous validation research and the theoretical conceptualization of cognitive fusion within ED psychopathology we hypothesized that the confirmatory factor analysis would result in a single-factor model for the CFQ-BI and that higher scores on the CFQBI would be significantly associated with lower levels of body image related psychological flexibility and greater ED symptom severity. Due to the exploratory nature of our measurement invariance analyses, no specific hypotheses were made.

\section{Methods}

\section{Participants}

Participants included 175 adolescent and adult females admitting to Avalon Hills Eating Disorder Specialists, a for-profit residential ED treatment facility for adolescent and adult women between November 2015 and June 2020. The sample for the current study included individuals admitting to both adolescent (age range $=11-17$ years, $n=75$ ) and adult (age range $=$ $n=100)$ residential units. At the time of admission, all participants completed a clinical 
interview with the masters or doctoral level clinician primarily assigned to their case throughout the course of treatment in order to determine the appropriate ED diagnosis as defined by the Diagnostic and Statistical Manual of Mental Disorders-Fifth Edition (DSM-5; American Psychiatric Association, 2013). Suggested diagnoses were then reviewed with an integrated treatment team based on intake reports generated by the clinician, registered dietitian, and medical director, before a formal diagnosis was given and approved by a doctoral level clinical psychologist (i.e., the clinical director). Table 1 provides descriptive statistics of the sample.

\section{Procedures}

All parts of the current study were approved by a university institutional review board. At the time of intake, all clients were informed about the opportunity to participate in research while receiving treatment and given details regarding what participation would entail. Clients and parents of adolescents under the age of 18 were informed that participation in the study was completely voluntary and that their choice to participate or not would have no impact on their clinical treatment. A total of 17 individuals/parents opted not to participate throughout the course of data collection. Those who consented to participation completed an online self-report assessment battery within the first three days following their intake. The assessment battery included demographic information, assessment of ED symptom severity, and a variety of other factors related to comorbid psychopathology, therapeutic processes, and targeted treatment outcomes. No compensation was provided to participants for data used in this study.

\section{Measures}

\section{Cognitive Fusion Questionnaire-Body Image (CFQ-BI; Ferreira et al., 2015)}

The CFQ-BI is a 10-item self-report measure designed to assess cognitive fusion related to body image. Responses are rated on a 7-point Likert-type scale $(1=$ Never True $; 7=$ Always 
True) to indicate how frequently each item applies to the individual. For the current study, the English translations of each item as worded within the original validation article (Ferreira et al., 2015) were utilized. Internal consistency of the CFQ-BI was excellent within the current study $($ Cronbach's alpha $=.97)$.

Body Image Acceptance and Action Questionnaire (BIAAQ; Sandoz et al., 2013)

The BIAAQ is a 12-item self-report measure of body image flexibility. Items are rated on a 7 -point Likert-type scale $(1=$ never true; 7 = always true $)$, with higher scores indicating greater levels of psychological inflexibility related to body image. The BIAAQ has been found to have good psychometric properties in both clinical (Lee et al., 2017) and nonclinical samples (Sandoz et al., 2013). In the current study the BIAAQ demonstrated excellent internal consistency $(\alpha=.91)$

Eating Disorder Examination Questionnaire (EDE-Q; Fairburn \& Beglin, 2008)

The EDE-Q was utilized as a measure of ED symptom severity. The EDE-Q is a selfreport measure of core attitudinal features and behaviors indicative of ED psychopathology. All items are responded to with regard to the individual's experience throughout the previous 28 days. 22 items are answered using 7-point Likert-type scale, with higher ratings reflecting greater levels of pathology. Six items assessing the frequency of various behaviors (e.g., binge eating, self-induced vomiting, driven exercise) are also included. The EDE-Q generates four subscale scores: eating restraint; eating concerns; shape concern; and weight concern and a global score reflecting overall ED symptom severity calculated by averaging the scores from each subscale ${ }^{1}$. In the current study, Cronbach's alpha was .92 for the global score, .88 for the

\footnotetext{
${ }^{1}$ Due to a researcher error, item 8 ("In the past 28 days how has thinking about shape or weight made it very difficult to concentrate on things you are interested in?) and item 25 ("In the past 28 days how dissatisfied have you been with your weight?) were omitted from the EDE-Q. However, missing data on the EDE-Q is notoriously common (Kelly, Lydecker, \& Mazzeo, 2017) and guidelines for handling missing data indicate that totals can still be
} 
restraint subscale, .79 for the eating concern subscale, .92 for the shape concern subscale, and .76 for the weight concern subscale. Only global scores were utilized for the analyses within the current study.

\section{Statistical analyses}

All analyses were conducted using RStudio (RStudio Team, 2020) based on R statistical software (R Development Core Team, 2017).

Confirmatory factor analysis (CFA) was conducted using the 'lavaan' package (Rosseel, 2012) to assess the single-factor structure of the CFQ-BI proposed by Ferreira et al. (2015) within a clinical sample. Model estimation was based upon the maximum likelihood (ML) method. Missing data were imputed with multiple imputation methods using the 'mice' package (van Buuren \& Groothuis-Oudshoorn, 2011) Only five item responses were missing from the CFQ-BI data among the entire sample.

Univariate coefficients of skewness $(S k)$ and kurtosis $(K u)$ were calculated using the 'MVN' package (Korkmaz et al., 2014) to assess the assumption of normality. Both Sk (range = -..37 [item 7]) to -1.12 [item 5]) and $K u($ range $=2.16$ [item 7] to 3.35 [item 3]) were below the identified critical values of $S k>|2|$ and $K u>|7|($ Curran et al., 1996; Ryu, 2011) indicating that ML methods were appropriate for model estimation. In addition, Mardia's multivariate skewness and kurtosis coefficients were calculated (948.49 \& 26.69, respectively) and indicated significant multivariate nonnormality $(p ’ s<.001)$. As such, the mean-and-variance-corrected

computed for the EDE-Q and each individual subscale if at least half of a given subscale's items are completed (Fairburn \& Beglin, 2008). It should also be noted that more recent psychometric evaluation of the EDE-Q suggests limited support for the original, theoretically derived, four factor structure and scoring procedures, however due to the lack of consensus and current data supporting an alternative factor structure specifically within clinical samples and the sole use of this measure for validation purposes, we chose to use the guidelines set forth by Faiburn \& Beglin (2008) for the current study. The two subscales impacted by the omission of these items (shape concern and weight concern) still each contained $>50 \%$ of their included items (shape concern $=87.5 \%$; weight concern $=60 \%$ ). Based on these scoring guidelines, we therefore chose to include these subscales in the analyses for the current study, pending no additional items were missing. 
(MLMV) robust variant of the maximum likelihood estimator was utilized for each model (Brosseau-Liard et al., 2012; Savalei, 2018). See Table 2 for item-level statistics. Goodness-offit for the model was evaluated using the normed chi-square $(\chi 2 / \mathrm{df}$, values $<5$ considered acceptable), the comparative fit index (CFI, values $\geq .95$ considered good and values $>.90$ considered acceptable), the root-mean-square error of approximation (RMSEA, values $<.08$ considered acceptable) with its $90 \%$ confidence interval, and the standardized root mean squared residual (SRMR, values $<.05$ considered good and values $<.08$ deemed acceptable) (Hooper et al., 2008). We also examined the Akaike's information criterion (AIC) and Schwarz's Bayesian information criterion (BIC) when comparing estimated models to evaluate model parsimony and efficiency in representing the data. Although specific cutoffs have not been defined for AIC or BIC values, smaller values are indicative of a more parsimonious model.

Multigroup analyses were then conducted using the best fitting model to test potential measurement invariance of the CFQ-BI between adolescents and adults and then between restrictive and binge-purge ED diagnoses. We followed the stepped procedure outlined by Widaman and Reise (1997) to examine whether configural (i.e., invariance of model form between groups), metric (i.e., invariance in item contributions to the latent construct between groups), and/or scalar (i.e. equivalence of item intercepts between groups) existed when using the CFQ-BI. To test for configural invariance (step 1) we examined whether the model the same pattern of free and fixed loadings for each group. If configural invariance was confirmed, we moved to step 2 and tested for metric invariance by constraining factor loadings in the model to be equivalent in the two groups being compared. Liklihood-Ratio test comparisons of the constrained model and the configural invariance model (from step 1), with insignificant results of this test indicating metric invariance is supported. If metric invariance was supported, we then 
moved to step 3 testing scalar invariance by adding additional constraints requiring item intercepts to be equivalent in the two groups being compared to the constrained model from step

2. This further constrained model was then compared with the constrained model from step 2 using Liklihood-Ratio tests, with insignificant results indicating that scalar invariance is supported. If support for all three forms of invariance is found, it is deemed appropriate to compare the means of latent construct (i.e., CFQ-BI scores) between groups. Therefore, if support was found for all three forms of invariance, independent sample t-tests were conducted to compare the mean scores on the CFQ-BI.

\section{Results}

\section{Confirmatory Factor Analysis}

In the single-factor model, the indicators all showed significant positive factor loadings, with standardized coefficients ranging from .777 to .938 ( $p$ 's $<.001)$. Fit indices for the model varied, representing a poor to mediocre fit to the data, $\chi^{2} / d f=2.16, p<.001 ; \mathrm{CFI}=.85$; RMSEA $=.08,90 \% \mathrm{CI}$ upper $=.105$, lower $=.055 ; \mathrm{SRMR}=.043$.

We reviewed residual correlations and modification indices to determine whether including additional parameters in the model may improve model fit. The largest modification index $(\mathrm{mi}=61.57)$ indicated that the model fit would be improved if the error terms of item 6 ("My thoughts regarding my body image distract me from what I'm actually doing") and item 7 ("I get so caught up in my thoughts about my physical appearance that I am unable to do the things that I most want to do") were permitted to covary. This was also consistent with the large residual correlation (coefficient $=.188$ ) observed between these variables. Upon reviewing the measure, we determined that these two items are the only items that describe the aspect of cognitive fusion where entanglement in thoughts elicits perceived behavioral problems as 
opposed to internal experiences of distress for respondents. Thus, it was unsurprising that these items demonstrated residual covariance beyond that observed among the ten items of the CFQ$\mathrm{BI}$ as a whole. Considering the theoretical notion that cognitive fusion manifests as an entanglement with one's thoughts such that they drive one's behavioral choices, we made a post hoc model modification allowing these two items to covary.

We re-ran the confirmatory factor analysis with the modified model. All indicators once again showed significant positive factor loadings. Additionally, all fit indices showed values representing acceptable fit: $\chi^{2} / d f=1.78, p<.001 ; \mathrm{CFI}=.90 ; \mathrm{RMSEA}=.067,90 \% \mathrm{CI}$ upper $=$ .094 , lower $=.038 ;$ SRMR $=.034$. We also reviewed the AIC and BIC values for the modified model in comparison to the original model. Both values were lower for the modified model $(\mathrm{AIC}=4806.528, \mathrm{BIC}=4872.989)$ relative to the original model $(\mathrm{AIC}=4873.16, \mathrm{BIC}=$ 4936.455), indicating the modified model provided a more efficient representation of the data. Based on the consistency of improved model fit and efficiency, the modified model was retained for multigroup analyses.

\section{Multigroup Analyses}

Multigroup analyses of the modified model were also conducted to test measurement invariance across adolescents and adults, with fit indices for each model presented in Table 3. We first ran a CFA of the model within each group, with all fit indices denoting adequate fit and a consistent pattern of free and fixed loadings in each group, supporting the presence of configural invariance. We then evaluated metric invariance $\left(\Delta \chi^{2}=6.80, p=.66\right)$ and scalar invariance $\left(\Delta \chi^{2}=11.24, p=.26\right)$ models using Likelihood-Ratio tests, with non-significant changes in $\chi^{2}$ supporting both. These findings indicated no evidence that the CFQ-BI assesses body image-related cognitive fusion differently for adolescents and adults and that it was 
appropriate to compare the mean of adolescent scores $(M=46.89, S D=15.19)$ on the CFQ-BI to that of adults $(M=57.05, S D=13.24)$. An independent samples t-test was conducted and a significant difference was found $\left(t_{(173)}=-4.72, p<.001\right)$ such that adolescents reported significantly lower levels of body image related cognitive fusion compared to adults.

Multigroup analyses were also conducted using the modified model to compare measurement invariance across individuals with ED diagnoses classified by binge-eating/purging behaviors with those classified with ED diagnoses indicating restrictive behaviors only (See Table 3 for fit indices). Individuals diagnosed with Anorexia Nervosa-Restrictive Type (AN-R) and Avoidant/Restrictive Food Intake Disorder (ARFID) were included in the "restrictive behaviors" group $(n=89)$ and individuals diagnosed with Anorexia Nervosa - Binge/Purge Type (AN-BP), Bulimia Nervosa (BN), and Binge Eating Disorder (BED) were included in the "binge-eating/purging behaviors" group $(n=84)$. The two participants in the sample diagnosed with Other Specified Feeding and Eating Disorder (OSFED) were not included in these analyses due to a lack of information on specific behavioral presentations warranting their diagnosis.

Once again, configural invariance of the model was supported. Additionally, metric $\left(\Delta \chi^{2}=7.09\right.$, $p=.63)$ and scalar invariance $\left(\Delta \chi^{2}=9.90, p=.36\right)$ were supported. The group mean of individuals with diagnoses classified by binge/purge behaviors $(M=55.53, S D=14.67)$ were then compared to that of individuals with diagnoses classified by restrictive behaviors $(M=$ 49.96, $S D=14.76)$ using an independent samples t-test. Once again a significant difference was found $\left(t_{(173)}=2.50, p=.01\right)$ indicating that individuals with disorders classified by binge/purge behaviors report levels of body image related cognitive fusion that are significantly greater than individuals with diagnoses classified by restrictive behaviors.

\section{Construct Validity}


To determine construct validity, we used Pearson correlations to compare average scores on the CFQ-BI $(M=52.41, S D=14.84)$ with those on the BIAAQ $(M=60.01, S D=16.08)$ and the EDE-Q $(M=3.91, S D=1.56)$. CFQ-BI scores were significantly associated with scores on the BIAAQ $(r=.76, p<.001)$ such that individuals who report greater body image related psychological inflexibility also report higher levels of body image related cognitive fusion. Similarly, scores on the CFQ-BI were significantly correlated with scores on the EDE-Q ( $r=.78$, $p<.001$ ), such that individuals who reported greater ED severity also reported higher levels of body image related cognitive fusion.

\section{Incremental Validity}

Incremental validity was examined for CFQ-BI scores over a measure of body image related psychological flexibility (BIAAQ) using partial correlations. Specifically, we controlled for body image related psychological flexibility on the relationship between CFQ-BI scores and ED severity. Results indicated that CFQ-BI scores were still significantly positively associated with ED severity $(r=.56, p<.001)$ after controlling for body image related psychological flexibility.

\section{Discussion}

The current study evaluated the psychometric properties of the CFQ-BI, a measure designed to evaluate body image-related cognitive fusion, when utilized in a clinical sample. Specifically, we examined the psychometric properties of the original single-factor structure of the CFQ-BI when used with a sample of English-speaking adolescents and adults admitting to residential treatment for an ED and evaluated its convergent and incremental validity. We also evaluated measurement invariance when using the CFQ-BI with different age groups (i.e. 
adolescents or adults) and across different ED behavioral presentations (i.e. restrictive or binge/purge behaviors).

Our results confirmed the single-factor structure of the CFQ-BI described in the initial validation study (Ferreira et al., 2015), but the model demonstrated poor fit with our sample. A modified single-factor model structure based on the theory-driven examination of modification indices that included an additional parameter allowing the residuals of items 6 and 7 to covary resulted in a more acceptable fit. When reviewing these items prior to making this post-hoc modification, we noted these were the only two items included in the measure that addressed the specific impact one's entanglement with their body image related thoughts has on their behaviors. While the results of the CFA suggested that the modified model demonstrated acceptable fit within our sample, the impact of this additional parameter warrants further consideration. It is possible that the additional variance among the residuals of these two items above and beyond that observed between any of the items may be indicative of multidimensionality within the latent construct of body image related fusion. Specifically, if our theoretical interpretation of the difference between these items as capturing the behavioral impact of entanglement with body image related thoughts is accurate, it is possible that body image related fusion may actually be better defined as a multi-factor construct (e.g., having distinct factors defined for emotional distress and behavioral control). Future research is therefore needed to determine if the correlation between these items appears to be benign residual variance, or whether a new or amended version of the CFQ-BI containing a broader assessment of the behavioral factor is warranted.

Our analyses of structural invariance indicated that the modified single-factor structure of the CFQ-BI remained consistent between adolescents and adults diagnosed with EDs and 
between individuals engaging primarily in restrictive disordered eating behaviors relative to those engaging primarily in binge and/or purging behaviors. Specifically, configural, metric, and scalar invariance was supported in both sets of group comparisons, indicating that the CFQ-BI appears to measure the latent factor of body image related cognitive fusion consistently across groups, and that the individual items appear to operate similarly within each group. The support for measurement invariance at each of these levels suggests that scores on CFQ-BI may be interpreted consistently across age and behavioral groups and that it was appropriate to statistically compare the scores between groups on this measure in the current study.

We found that the adolescents in our study reported significantly less body image related cognitive fusion relative to adults. While this finding suggests that differences in levels of body image related cognitive fusion may exist between age groups, our analyses are simply associative so conclusions as to why this difference exists cannot be made. However, this finding warrants future research to determine whether this finding is replicated in larger clinical samples and to explore potential mechanisms underlying this difference. For example, future studies might explore the hypothesis that cognitive fusion increases over time, resulting in age possessing a linear relationship with CFQ-BI scores. Preliminary research in flexible learning and hypothesis adaptation provides a basis for this hypothesis, highlighting that as one grows older, they appear to become "less flexible" and tend to prefer buying into familiar hypotheses, even when presented with new information inconsistent with these hypotheses (Gopnik et al., 2017). Alternatively, it may be that adult clients have struggled with their ED symptoms longer than adolescent clients, and that the duration of ED symptoms is what accounts for the observed difference between these two groups. While these may be two plausible hypotheses, our findings do not suggest any specific underlying reason for the significant difference in adult and 
adolescent CFQ-BI scores, and the observed difference in the current study presents an area for future exploration.

We also found that individuals primarily engaging in restrictive disordered eating behaviors reported significantly less body image related cognitive fusion compared to those primarily engaging in binge and/or purge behaviors. Once again, this finding is the result of exploratory comparison analyses therefore no causal conclusion can be made as to why this difference emerged. However, this finding also presents directions for future research. Specifically, future studies should explore whether body image related fusion is predictive of specific ED behaviors, and if so, if it has greater predictive value for certain behaviors moreso than others. It may also be that those individuals engaging in binge/purge presentations who are seeking treatment at a residential level of care tend to experience more severe psychological symptoms broadly that necessitate this level of intervention, as opposed to physical symptoms (e.g., low BMI; malnourishment) that may more often necessitate residential care for individuals engaging in restrictive behaviors. Although it is the case that in the current study the restrictive behaviors group did indeed have a lower average BMI relative to the binge/purge group, recent research suggests that lower BMI is frequently not associated with more severe ED symptomology (Grilo et al., 2015; Machado et al., 2017). It is therefore encouraged that future research examine whether CFQ-BI scores appear to be uniquely related to certain physical, psychological, and behavioral symptoms differentially.

Future studies should also explore whether varying degrees of a third factor may influence this pattern within and across specific ED diagnoses. For example, research findings suggest that individuals who engage in binge/purge behaviors report higher levels of impulsivity and affective urgency (i.e., a tendency to act impulsively when experiencing heightened positive 
or negative affect) relative to individuals who engage in primarily restrictive behaviors.

Theoretically, one may posit that impulsivity or non-acceptance of emotions may mediate the relationship between levels of body image related cognitive fusion within ED symptoms transdiagnostically. If so, the tendency for these factors to be particularly elevated among individuals who engage in binge/purge behaviors may explain the higher scores observed in our study. Alternatively, it may be that the functionality of specific disordered eating behaviors explains their relationship with body image related cognitive fusion. For example, findings from a recent study suggest that restrictive behaviors appear to primarily serve automatic positive reinforcement functions (Wang et al., 2020), whereas binge eating, and purging have been found to be predominantly maintained by automatic negative reinforcement (Wedig \& Nock, 2010). Once again, the findings from the current study solely denote that differences may exist, but present possible directions for future research.

The current study is not without limitations. The generalizability of our results is limited in several ways. Our sample was composed only of female participants and was relatively homogeneous with regard to ethnicity. It was also a convenience sample made up of individuals seeking treatment at the same residential treatment facility, with all clients admitting to the facility eligible to participate. This is likely to have introduced heterogeneity in comorbid psychopathology. Further, over $80 \%$ of the sample diagnosed with either AN-R or AN-BP which is similar to the diagnostic distributions observed in other recent studies of residential ED samples (e.g. Fisher et al., 2020; Muzi et al., 2020). Therefore, while our findings may be generalizable to other clinical ED samples seeking intensive care, future research is needed to determine whether our findings would be replicated in solely bulimia nervosa and/or binge eating samples, as well as those not currently seeking treatment or being treated at lower levels 
of care that still meet clinical criteria for an ED diagnosis. Future research using the CFQ-BI among individuals with EDs should further examine the psychometric properties when differentiating between individuals with varying comorbid diagnoses (e.g., mood disorders vs. anxiety disorders) to determine if the factor structure observed in the current sample is upheld. Our also does not include a comparative sample of healthy controls to determine whether levels of body image related cognitive fusion appear significantly elevated in individuals with clinically significant EDs. Such research is needed to denote whether distinct elevations in body imagerelated cognitive fusion exist in clinical populations, and whether there appears to be a clinical "cutoff" or threshold that may differentiate individuals with or without ED pathology.

Our samples for measurement invariance analyses are also small which may increase the risk of Type I error and limit the robustness of our results. However, $\Delta \chi^{2}$ and fit indices appear to be fairly robust to sample size when using maximum likelihood estimators, and may actually be more susceptible to over-rejecting models that demonstrate good fit when samples are too large (Finch et al., 2018; Putnick \& Bornstein, 2016). Our findings consistently demonstrated acceptable measurement invariance therefore sample size may be less problematic in the current study. Nonetheless, replication with variable sample size is recommended to strengthen support of these findings. Similarly, our adolescent and adult samples were unequal $(n=75$ and $n=100$ respectively). Simulation research suggests that measurement invariance findings may be biased such that they are more likely to mask violations in invariance when groups are "severely" unbalanced, however within this study "severely unbalanced" was defined as a ratio difference of 1:2 or larger (Yoon \& Lai, 2018). Based on these limitations, readers should consider the measurement invariance analyses presented in the current study as preliminary and interpret them with this in mind. 
Although our modified model did demonstrate adequate fit, our use of modification indices also innately introduces an increased risk for overfitting the model to the data. This fact, combined with the poor fit observed using the previously validated model of the CFQ-BI suggests that further confirmatory research is warranted to determine whether the psychometrics of the CFQ-BI within clinical ED samples using the modified model are sustained. Additionally, our study's cross-sectional design prevents us from drawing any conclusions regarding temporal stability of the CFQ-BI's psychometrics in this population or about causality in terms of the relationships observed between included variables. As previously denoted, cognitive fusion is conceptualized as a psychological process thought to maintain a variety of psychopathology, and as a core treatment target of ACT. Therefore, future longitudinal studies examining whether levels of body image related cognitive fusion appear to change following ACT-based treatments for EDs and whether such changes are associated with other targeted outcomes is needed to evaluate the theoretical accuracy and efficacy of ACT for EDs. Lastly, the unintentional omission of two items on the EDE-Q introduces possible error in the ED severity scores used for comparison in the current study.

\section{Conclusion}

Our study is the first to our knowledge to evaluate the psychometric properties of the CFQ-BI in a clinically significant ED sample. It is also the first to evaluate these properties using the English version, within a western English-speaking sample. Overall, our findings indicate that the CFQ-BI appears to assess the process of body image-related cognitive fusion adequately and consistently within a clinical ED sample when the modified factor model allowing the residuals of items 6 and 7 to covary is used. Clinicians, especially those seeking to target cognitive fusion within treatment with clients experiencing clinically significant EDs may 
find this assessment particularly useful in understanding the severity of body image related fusion the client is struggling with in order to develop a complete and process-oriented case conceptualization. Our findings also provide support for using the CFQ-BI as a valid assessment tool in future research with ED samples, which is particularly useful considering its brevity and ease to administer. While our study provides support for the use of the CFQ-BI within individuals diagnosed with clinically significant EDs cross-sectionally, future research should seek to replicate these findings in more diverse clinical sample and to determine whether the CFQ-BI is an appropriate and sensitive assessment tool to evaluate temporal changes of body image-related fusion in response to therapeutic interventions targeting this process. Finally, future studies should seek to more directly examine the processes through which the relationship between CFQ-BI scores and ED behaviors may emerge to better inform etiological theories and intervention methods. 


\section{References}

American Psychiatric Association. (2013). Diagnostic and statistical manual of mental disorders (DSM-5®).

Barlow, D. H., Allen, L. B., \& Choate, M. L. (2004). Toward a unified treatment for emotional disorders. Behavior Therapy, 35(2), 205-230.

\section{https://doi.org/https://doi.org/10.1016/S0005-7894(04)80036-4}

Bento, S., Ferreira, C., Mendes, A. L., \& Marta-Simões, J. (2017). Emotion regulation and disordered eating: The distinct effects of body image-related cognitive fusion and body appreciation. Psychologica, 60(2), 11-25. https://doi.org/10.14195/1647-8606_60-2_1

Brosseau-Liard, P. E., Savalei, V., \& Li, L. (2012). An investigation of the sample performance of two nonnormality corrections for RMSEA. Multivariate Behavioral Research, 47(6), 904-930. https://doi.org/10.1080/00273171.2012.715252

Curran, P. J., West, S. G., \& Finch, J. F. (1996). The robustness of test statistics to nonnormality and specification error in confirmatory factor analysis. Psychological Methods, 1(1), 16. https://doi.org/10.1037/1082-989X.1.1.16

Duarte, C., \& Pinto-Gouveia, J. (2017). The impact of early shame memories in binge eating disorder: The mediator effect of current body image shame and cognitive fusion. Psychiatry Research, 258, 511-517. https://doi.org/10.1016/j.psychres.2017.08.086

Duarte, C., Pinto-Gouveia, J., \& Ferreira, C. (2017). Ashamed and fused with body image and eating: Binge eating as an avoidance strategy. Clinical Psychology \& Psychotherapy, 24(1), 195-202. https://doi.org/10.1002/cpp.1996 
Fairburn, C. G., \& Beglin, S. J. (2008). Eating Disorder Examination Questionnaire. In C. G. Fairburn (Ed.), Cognitive behavior therapy and eating disorders (pp. 309-313). Guilford Press.

Ferreira, C., Palmeira, L., \& Trindade, I. A. (2014). Turning eating psychopathology risk factors into action. The pervasive effect of body image-related cognitive fusion. Appetite, 80 , 137-142. https://doi.org/10.1016/j.appet.2014.05.019

Ferreira, C., \& Trindade, I. A. (2015). Body image-related cognitive fusion as a main mediational process between body-related experiences and women's quality of life. Eating and Weight Disorders-Studies on Anorexia, Bulimia and Obesity, 20(1), 91-97. https://doi.org/https://doi.org/10.1007/s40519-014-0155-y

Ferreira, C., Trindade, I. A., Duarte, C., \& Pinto-Gouveia, J. (2015). Getting entangled with body image: Development and validation of a new measure. Psychology and Psychotherapy: Theory, Research and Practice, 88(3), 304-316. https://doi.org/10.1111/papt.12047

Finch, H. W., French, B. F., \& Hernández Finch, M. E. (2018). Comparison of methods for factor invariance testing of a 1-factor model with small samples and skewed latent traits. Frontiers in Psychology, 9, 332. https://doi.org/https://doi.org/10.3389/fpsyg.2018.00332

Fisher, M., Henretty, J. R., Cox, S. A., Feinstein, R., Fornari, V., Moskowitz, L., Schneider, M., Levine, S., Malizio, J., \& Fishbein, J. (2020). Demographics and outcomes of patients with eating disorders treated in residential care. Frontiers in Psychology, 10, 2985. https://doi.org/https://doi.org/10.3389/fpsyg.2019.02985

Gillanders, D. T., Bolderston, H., Bond, F. W., Dempster, M., Flaxman, P. E., Campbell, L., Kerr, S., Tansey, L., Noel, P., \& Ferenbach, C. (2014). The development and initial 
validation of the Cognitive Fusion Questionnaire. Behavior Therapy, 45(1), 83-101. https://doi.org/10.1016/j.beth.2013.09.001

Gopnik, A., O’Grady, S., Lucas, C. G., Griffiths, T. L., Wente, A., Bridgers, S., Aboody, R., Fung, H., \& Dahl, R. E. (2017). Changes in cognitive flexibility and hypothesis search across human life history from childhood to adolescence to adulthood. Proceedings of the National Academy of Sciences, 114(30), 7892-7899. https://doi.org/https://doi.org/10.1073/pnas.1700811114

Grilo, C. M., Ivezaj, V., \& White, M. A. (2015). Evaluation of the DSM-5 severity indicator for binge eating disorder in a clinical sample. Behaviour Research and Therapy, 71, 110-114. https://doi.org/https://doi.org/10.1016/j.brat.2015.05.003

Hayes, S. C., Strosahl, K. D., \& Wilson, K. G. (2011). Acceptance and commitment therapy: The process and practice of mindful change (2 ed.). Guilford Press.

Hofmann, S. G., Asnaani, A., Vonk, I. J., Sawyer, A. T., \& Fang, A. (2012). The efficacy of cognitive behavioral therapy: A review of meta-analyses. Cognitive Therapy and Research, 36(5), 427-440. https://doi.org/10.1007/s10608-012-9476-1

Hofmann, S. G., \& Hayes, S. C. (2018). The history and current status of CBT as an evidencebased therapy. In S. C. Hayes \& S. G. Hofmann (Eds.), Process-based CBT: The science and core clinical competencies of cognitive behavioral therapy (1 ed., pp. 7-21). Context Press.

Hooper, D., Coughlan, J., \& Mullen, M. R. (2008). Structural equation modelling: Guidelines for determining model fit. The Electronic Journal of Business Research Methods, 6(1), 5360. https://doi.org/10.1080/16506073.2018.1513556 
Korkmaz, S., Goksuluk, D., \& Zararsiz, G. (2014). MVN: An R package for assessing multivariate normality. The R Journal, 6(2), 151-162. https://journal.rproject.org/archive/2014-2/korkmaz-goksuluk-zararsiz.pdf

Krafft, J., Haeger, J. A., \& Levin, M. E. (2019). Comparing cognitive fusion and cognitive reappraisal as predictors of college student mental health. Cognitive Behaviour Therapy, 48(3), 241-252.

Lee, E. B., Smith, B. M., Twohig, M. P., Lensegrav-Benson, T., \& Quakenbush-Roberts, B. (2017). Assessment of the Body Image-Acceptance and Action Questionnaire in a female residential eating disorder treatment facility. Journal of Contextual Behavioral Science, 6(1), 21-28. https://doi.org/10.1016/j.jcbs.2016.11.004

Lucena-Santos, P., Trindade, I. A., Oliveira, M., \& Pinto-Gouveia, J. (2017). Cognitive Fusion Questionnaire-Body Image: Psychometric properties and its incremental power in the prediction of binge eating severity. The Journal of Psychology, 151(4), 379-392. https://doi.org/doi.org/10.1080/00223980.2017.1305322

Machado, P. P., Grilo, C. M., \& Crosby, R. D. (2017). Evaluation of the DSM-5 severity indicator for anorexia nervosa. European Eating Disorders Review, 25(3), 221-223. https://doi.org/https://doi.org/10.1002/erv.2508

Manlick, C. F., Cochran, S. V., \& Koon, J. (2013). Acceptance and commitment therapy for eating disorders: Rationale and literature review. Journal of Contemporary Psychotherapy, 43(2), 115-122. https://doi.org/https://doi.org/10.1007/s10879-012-9223$\underline{7}$

McEvoy, P. M., Watson, H., Watkins, E. R., \& Nathan, P. (2013). The relationship between worry, rumination, and comorbidity: Evidence for repetitive negative thinking as a 
transdiagnostic construct. Journal of Affective Disorders 151(1), 313-320. https://doi.org/https://doi.org/10.1016/j.jad.2013.06.014

Merwin, R. M., Timko, C. A., Moskovich, A. A., Ingle, K. K., Bulik, C. M., \& Zucker, N. L. (2010). Psychological inflexibility and symptom expression in anorexia nervosa. Eating Disorders, 19(1), 62-82. https://doi.org/10.1080/10640266.2011.533606

Morris, L., \& Mansell, W. (2018). A systematic review of the relationship between rigidity/flexibility and transdiagnostic cognitive and behavioral processes that maintain psychopathology. Journal of Experimental Psychopathology, 9(3), 2043808718779431. https://doi.org/10.1177/2043808718779431

Muzi, L., Tieghi, L., Rugo, M. A., \& Lingiardi, V. (2020). Evaluating empirically valid and clinically meaningful change in intensive residential treatment for severe eating disorders at discharge and at a 6-month follow-up. Eating and Weight Disorders-Studies on Anorexia, Bulimia and Obesity, 25(6), 1609-1620. https://doi.org/https://doi.org/10.1007/s40519-019-00798-2

Pinto-Gouveia, J., Dinis, A., Gregório, S., \& Pinto, A. M. (2020). Concurrent effects of different psychological processes in the prediction of depressive symptoms-the role of cognitive fusion. Current Psychology, 39(2), 528-539. https://doi.org/https://doi.org/10.1007/s12144-017-9767-5

Putnick, D. L., \& Bornstein, M. H. (2016). Measurement invariance conventions and reporting: The state of the art and future directions for psychological research. Developmental Review, 41, 71-90. https://doi.org/https://doi.org/10.1016/j.dr.2016.06.004

R Development Core Team. (2017). R: A language and environment for statistical computing. In R Development Core Team Vienna, Austria. https://www.R-project.org/ 
Rosseel, Y. (2012). lavaan: An R package for structural equation modeling. Journal of Statistical Software, 48(2), 1-36. http://www.jstatsoft.org/v48/i02/

RStudio Team. (2020). RStudio: Integrated development for R. In RStudio, PBC. http://www.rstudio.com

Ryu, E. (2011). Effects of skewness and kurtosis on normal-theory based maximum likelihood test statistic in multilevel structural equation modeling. Behavior Research Methods, 43(4), 1066-1074. https://doi.org/10.3758/s13428-011-0115-7

Sandoz, E. K., Wilson, K. G., Merwin, R. M., \& Kellum, K. K. (2013). Assessment of body image flexibility: The Body Image-Acceptance and Action Questionnaire. Journal of Contextual Behavioral Science, 2(1-2), 39-48. https://doi.org/10.1016/j.jcbs.2013.03.002

Savalei, V. (2018). On the computation of the RMSEA and CFI from the mean-and-variance corrected test statistic with nonnormal data in SEM. Multivariate Behavioral Research, 53(3), 419-429. https://doi.org/10.1080/00273171.2018.1455142

Scardera, S., Sacco, S., Di Sante, J., \& Booij, L. (2020). Body image-related cognitive fusion and disordered eating: the role of self-compassion and sad mood. Eating and Weight Disorders-Studies on Anorexia, Bulimia and Obesity, 1-8. https://doi.org/10.1007/s40519-020-00868-w

Trindade, I. A., \& Ferreira, C. (2014). The impact of body image-related cognitive fusion on eating psychopathology. Eating Behaviors, 15(1), 72-75. https://doi.org/10.1016/j.eatbeh.2013.10.014

van Buuren, S., \& Groothuis-Oudshoorn, K. (2011). mice: Multivariate Imputation by Chained Equations in R. Journal of Statistical Software, 45(3), 1-67. 
Wang, S., Fox, K., Boccagno, C., Hooley, J., Mair, P., Nock, M., \& Haynos, A. (2020).

Functional assessment of restrictive eating: A three-study transdiagnostic investigation. PsyArxiv. https://doi.org/10.31234/osf.io/q2ezw

Wedig, M. M., \& Nock, M. K. (2010). The functional assessment of maladaptive behaviors: A preliminary evaluation of binge eating and purging among women. Psychiatry Research, 178(3), 518-524. https://doi.org/https://doi.org/10.1016/j.psychres.2009.05.010

Widaman, K. F., \& Reise, S. P. (1997). Exploring the measurement invariance of psychological instruments: Applications in the substance use domain. In K. J. Bryant, M. Windle, \& S. G. West (Eds.), American Psychological Association https://doi.org/https://doi.org/10.1037/10222-009

Yoon, M., \& Lai, M. H. (2018). Testing factorial invariance with unbalanced samples. Structural Equation Modeling: A Multidisciplinary Journal, 25(2), 201-213.

https://doi.org/https://doi.org/10.1080/10705511.2017.1387859 


\section{Table 1}

Descriptive Statistics

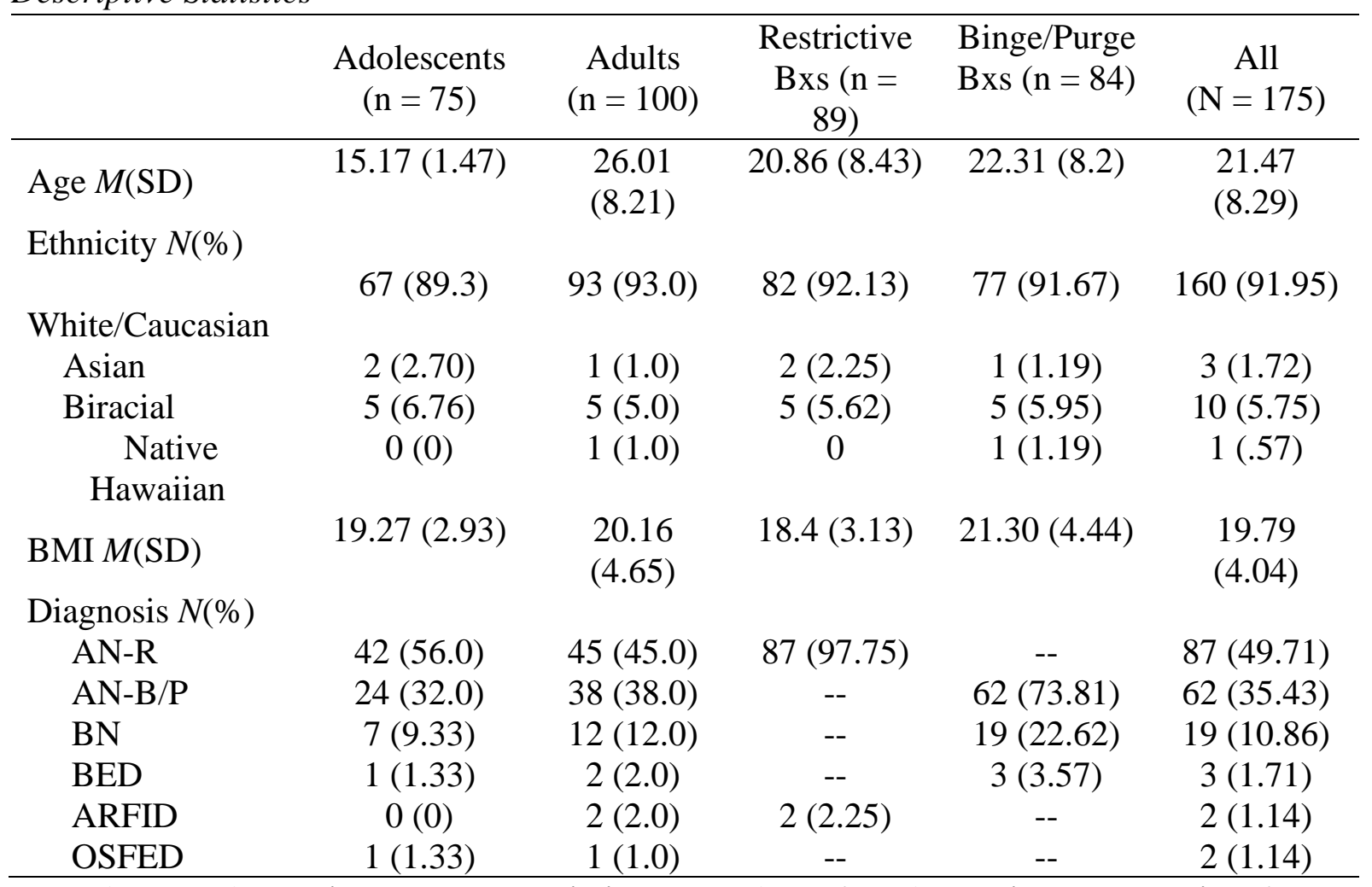

Note: $\mathrm{AN}-\mathrm{R}=$ Anorexia Nervosa-Restrictive Type; AN-B/P = Anorexia Nervosa-Binge/Purge

Type; BN = Bulimia Nervosa $;$ BED = Binge Eating Disorder; ARFID = Avoidant and

Restrictive Food Intake Disorder; OSFED = Other Specified Feeding and Eating Disorder 
Table 2

Cognitive Fusion Questionnaire-Body Image Item-Level Statistics

\begin{tabular}{|c|c|c|c|c|c|}
\hline & $M(S D)$ & $S k$ & $K u$ & $\begin{array}{l}\text { Corrected } \\
\text { item-total } \\
\text { correlation }\end{array}$ & $\begin{array}{l}\alpha \text { if item } \\
\text { deleted }\end{array}$ \\
\hline $\begin{array}{l}\text { 1. My thoughts relating to my body } \\
\text { image cause me distress or } \\
\text { emotional pain. }\end{array}$ & $5.51(1.55)$ & -0.95 & 3.22 & .84 & .97 \\
\hline $\begin{array}{l}2 . \text { I tend to get very entangled in my } \\
\text { thoughts concerning my body or } \\
\text { body image. }\end{array}$ & $5.50(1.61)$ & -0.99 & 3.22 & .90 & .96 \\
\hline $\begin{array}{l}\text { 3. I feel upset when I have negative } \\
\text { thoughts about my body (or physical } \\
\text { appearance). }\end{array}$ & $5.61(1.56)$ & -1.01 & 3.35 & .81 & .97 \\
\hline $\begin{array}{l}\text { 4. I get very focused on distressing } \\
\text { thoughts about my body image. }\end{array}$ & $5.26(1.68)$ & -0.77 & 2.72 & .92 & .96 \\
\hline $\begin{array}{l}\text { 5. It's such a struggle to let go of } \\
\text { upsetting thoughts about my body } \\
\text { shape even when I know that letting } \\
\text { go would be helpful. }\end{array}$ & $5.46(1.76)$ & -1.12 & 3.32 & .83 & .97 \\
\hline $\begin{array}{l}\text { 6. My thoughts regarding my body } \\
\text { image distract me from what I'm } \\
\text { actually doing. }\end{array}$ & 4.79 (1.77) & -0.52 & 2.34 & .85 & .97 \\
\hline $\begin{array}{l}\text { 7. I get so caught up in my thoughts } \\
\text { about my physical appearance that I } \\
\text { am unable to do the things that I } \\
\text { most want to do. }\end{array}$ & $4.46(1.86)$ & -0.36 & 2.16 & .78 & .97 \\
\hline $\begin{array}{l}\text { 8. I over-analyze my physical } \\
\text { appearance or my body shape to the } \\
\text { point where it's unhelpful to me. }\end{array}$ & $5.40(1.73)$ & -1.03 & 3.18 & .86 & .97 \\
\hline $\begin{array}{l}\text { 9. I struggle with my thoughts } \\
\text { related to my body or physical } \\
\text { appearance. }\end{array}$ & $5.65(1.62)$ & -1.10 & 3.33 & .88 & .96 \\
\hline $\begin{array}{l}\text { 10. Once I've thought about my } \\
\text { body or body shape in an upsetting } \\
\text { way it's difficult for me to focus on } \\
\text { anything else. }\end{array}$ & $5.04(1.77)$ & -0.76 & 2.66 & .89 & .96 \\
\hline Total & $\begin{array}{l}52.70 \\
(14.94)\end{array}$ & -0.86 & 2.86 & -- & -- \\
\hline
\end{tabular}


Table 3

Fit Indices for Measurement Invariance Models

\begin{tabular}{lcccccc}
\hline & \multicolumn{1}{c}{$\chi^{2}(\mathrm{df})$} & CFI & RMSEA & SRMR & AIC & BIC \\
\hline Adolescent and Adult Models & & & & & \\
Configural & $199.45(68)$ & .96 & .04 & .04 & 4709.61 & 4905.83 \\
Metric & $205.24(77)$ & .96 & .04 & .05 & 4697.41 & 4865.14 \\
Scalar & $217.63(86)$ & .95 & .04 & .06 & 4691.80 & 4831.05 \\
& & & & & & \\
Restrictive and Binge/Purge Models & & & & & \\
Configural & $209.72(68)$ & .91 & .06 & .04 & 4835.49 & 5031.71 \\
Metric & $216.32(77)$ & .90 & .06 & .05 & 4824.09 & 4991.82 \\
Scalar & $226.48(86)$ & .90 & .06 & .05 & 4816.25 & 4955.50 \\
\hline
\end{tabular}

